\title{
A planetary perspective on the deep Earth
}

\author{
David J. Stevenson
}

\section{Earth's composition, evolution and structure are in part a legacy of provenance (where it happened to form) and chance (the stochastics of that formation).}

Earth is an engine, tending to obliterate some of the evidence of events that are distant in time, but a memory is retained in its chemistry, its isotopes, the presence of the Moon, perhaps also in geophysical observables such as the temperature of the core and the nature of the mantle immediately above the core, and maybe even in the existence of plate tectonics and life. The remarkable growth in the study and understanding of Earth has happened in parallel with a spectacular era of planetary exploration, relevant astronomical discoveries and computational and theoretical advances, all of which help us to place Earth and its interior in a perspective that integrates the Earth sciences with extraterrestrial studies and basic sciences such as condensed-matter physics. However, progress on the biggest challenges in understanding the deep Earth continues to rely mainly on looking down rather than looking up.

\section{A planetary perspective}

Earth is a planet - one of many. There is nothing particularly remarkable about our home, except perhaps that it is suitable for life like us - arguably a tautology. It happens to be the largest of its type in the Solar System, but as there are only three others of the terrestrial type (Mercury, Venus and Mars) this is not particularly significant. Among planets in general, it is small.

In the past decade, we have seen an astonishing explosion in our catalogue of planets outside the Solar System to about 250 so far (see the Extrasolar Planets Encyclopaedia, http://exoplanet.eu/ catalog.php). These are mostly planets that we suspect are like Jupiter, very different from Earth. But as time goes on and detection methods improve, we can expect to find bodies that are Earth-like at least to the extent of being made predominantly of rock and iron, the primary constituents of our planet. Some would claim we might already be finding such bodies ${ }^{1}$, initially those that are more massive than Earth.

If planets were like atoms or molecules, or even crystals, we could speak of their characteristics (their DNA, so to speak) in a very compact way, just as a handbook might list the properties of a material. Planets are richer, more complex and more resistant to reductionist thinking. Genetics is the science of heredity and variation in biological systems. By analogy, we can speak of the genetics of a planet such as Earth, while also acknowledging that environment has a role in its evolution and its current state.

Cosmologists are familiar with thinking about time logarithmically: a lot happened in a very short period of time back near the Big Bang. To some extent, it helps to think about planet formation in a similar way (Fig. 1). The events that defined Earth's formation and the initial conditions for its subsequent evolution are squeezed into an epoch that may have already been over within 100 million years of the formation of the Solar System. In this epoch more happened inside Earth and more energy was dissipated from within the planet than throughout all of subsequent geological time. We have no direct geological record of this earliest epoch in the form of rocks and must rely instead on other sources of evidence.

\section{Making planets}

Our understanding of planet formation involves four major inputs: astronomical observations of places where planetary systems may currently be forming, the study of meteorites that formed even before the epoch in which the Solar System's planets formed, study of the planets themselves (Earth among them), and theoretical modelling. None of these is very complete or satisfying. The astronomical observations tell us about disks and dust and only indirectly about possible planets, the meteorites come from parent bodies that were probably always in orbits beyond Mars and are not necessarily representative of Earth's building blocks, Earth itself is good at concealing its history (through frequent surface rejuvenation), and theory is often either too permissive (many adjustable parameters) or falls short of a correct description of process. Even so, a picture emerges that has undergone considerable testing and refinement in recent years.

Current models of planetary formation ${ }^{2-6}$ have had some success in explaining observations and have the following features. Almost 4.6 billion years ago, an interstellar cloud of gas and dust collapsed under the action of gravity. Angular momentum guaranteed that the collapse would be into a disk around the forming star (the Sun) rather than merely into the Sun alone. This disk had a radius of perhaps 50 astronomical units (AU), where $1 \mathrm{AU}$ is the distance between Earth and the Sun. Almost all the mass of this disk was outwards of the eventual orbit of Earth. The particular mix of elements was nothing unusual, having been set by nucleosynthesis for the heavy elements and the outcome of the Big Bang for the lightest elements. Conversion of the gravitational energy of infall into heat assured that temperatures would be high in the inner part of the disk, sufficient to vaporize much of the infalling dust. Subsequent cooling allowed the formation of dust embedded in the primarily hydrogen gas. Through gentle collisions, these particles aggregated into larger particles up to a centimetre or more in size.

Meteoritic evidence strongly indicates the formation of larger 'planetesimals' that were kilometres or more in size, on a timescale of less than a million years. This process is poorly understood: planetesimals may have arisen through gentle collision and sticking of smaller grains or they may have arisen through gravitational instabilities in the disk. Such processes are presumed to have occurred throughout the Solar System. The timing of formation of these bodies is well established at around 4,567 million years ago, and their collapse from the interstellar medium can only have occurred a million years or less before this because of evidence for the presence of short-lived radioactive elements. This precisely determined date therefore well defines the origin of the Solar System.

Almost a billion bodies $10 \mathrm{~km}$ in diameter would be needed to make an Earth. However, it is not thought likely that planetesimals were the actual building blocks of Earth. A dense swarm of such bodies in nearly circular low-inclination orbits is gravitationally unstable on a short timescale. In less than a million years, much larger bodies ('planetary embryos') are formed that are Moon- to Mars-sized. These arise because of gravitational focusing of impacts between bodies with low relative velocity. Outward from the asteroid belt, these embryos may 


\begin{tabular}{|c|c|c|}
\hline & & \\
\hline & - Planetesimals form & - Solar nebula eliminated \\
\hline $\begin{array}{l}\text { Collapse of an } \\
\text { interstellar cloud to } \\
\text { form the solar nebula }\end{array}$ & $\begin{array}{l}\text { - Moon-to-Mars-sized } \\
\text { embryos form rapidly } \\
\text { after this }\end{array}$ & $\begin{array}{l}\text { - Substantial proto-Earth } \\
\text { (more than } 50 \% \text { of final } \\
\text { mass) might already exist }\end{array}$ \\
\hline
\end{tabular}

$1 \mathrm{Myr}$

$10 \mathrm{Myr}$

Figure 1 A logarithmic view of the time of planetary formation. The left end corresponds to the initiation of a collapse to form the solar nebula, and is close to 4,567 million years ago. Much happened in the 1 to 100 million

exceed Earth in mass, but in the terrestrial zone they are still well short of Earth's final mass. This means we must build Earth from a modest number (100 or fewer) of these embryos, but adding in a sprinkling of planetesimals. The aggregation of embryos to even bigger bodies takes far longer than their formation, extending from tens of millions of years to as much as 100 million years, because it requires the excitation of eccentric orbits so that the embryos have an opportunity to collide ${ }^{3-5}$.

Earth and its companion terrestrial planets are a tiny part of the Solar System and it should come as no surprise that the presence of the giant planets, especially Jupiter, the most massive and closest of these to Earth, would have a role in Earth's formation. Jupiter must have formed while the hydrogen-dominated gas of the solar nebula was mostly still present $t^{6}$, and astronomical observations suggest that the gas may have been present in sufficient abundance for about 5 million years at most. We could perhaps imagine that the formation of Earth postdated the formation of Jupiter, and some models are of this kind. Realistically, a full understanding of Earth's formation probably requires a full understanding of Jupiter's formation. Jupiter is enriched in heavy elements relative to the Sun, and some part of that enrichment is likely to be present as a core. It is likely, although not certain, that this core was formed first, with the gas then placed on top. But whichever story is correct, the formation of Jupiter involves much more than the physics involved in building bodies such as Earth because we must understand gas accretion as well as the accretion of solids. At present, this understanding is incomplete. Models of Earth accretion are in many ways much more detailed than models of giant-planet formation, but they are contingent on understanding Jupiter.

\section{Planetary embryology}

We have evidence about some of the planetesimals because they are the presumed source of most meteorites, but the much larger embryos have not left direct evidence of their existence. Nonetheless, it is likely that their properties are important for understanding Earth. They formed so quickly that they probably partly melted, owing to the presence of the short-lived radioactive isotope ${ }^{26} \mathrm{Al}$. They may even have been big enough to undergo melting by the conversion of gravitational energy of formation into heat. Partial melting can be expected to cause the separation of a liquid iron alloy from the partly molten silicate mantle, and these embryos may even have had atmospheres. In short, they are planets with iron cores, short-lived but possessing properties derived from planetary processes rather than the properties of the precursor planetesimals. These differences from planetesimals can arise in a number of ways: ingassing (the incorporation of solar nebula gas, should the surface of the embryo be molten), the role of pressure (the mineral phases within the embryo and its crust can be different from those in a low-pressure planetesimal because of self-gravity), and the loss of material by escape (either because of high temperatures or through collisions). Close encounters, tidal disruption and the creation of debris during collisions are processes that are not currently well incorporated into models of planet formation.

The embryos responsible for forming Earth were not - indeed could not have been - built from planetesimals that formed at $1 \mathrm{AU}$, because the coalescence of the embryos necessarily requires their scattering around the inner part of the Solar System ${ }^{3-5}$. It is therefore incorrect to think of Earth's provenance and composition as being precisely defined,
- Last giant impact occurs,
plausibly lunar forming
- Earth's surface rapidly cools after this impact

\author{
- Life originates \\ - Rock record (cratons) \\ develops
}
100 Myr
1,000 Myr
Present

years (Myr) immediately after this, and the logarithmic scale correctly emphasizes the importance of this 100-Myr period, despite the shortness of this period compared with Earth's age.

and different from, say, those of Venus. On the other hand, some differences are expected purely by chance and, importantly, it is thought unlikely that any of the Earth-forming embryos formed out at locations where water ice could condense. Indeed, Earth is relatively dry, at least for the water inventory that we can measure (the oceans and upper mantle), and our water may have arisen through water-bearing planetesimals coming from greater distances rather than through water incorporated in the primary embryos. This remains somewhat controversial, and one of the goals of Earth science is to get a better understanding of Earth's complete water budget.

\section{Giant impacts and lunar formation}

The likely dominance of the embryos as building-blocks for Earth implies the predominance of giant impacts. We should not think of Earth's formation as the steady accumulation of mass but rather as a series of infrequent, highly traumatic events separated by periods of cooling and healing. The largest, and possibly the last, of these events is thought to have been responsible for the formation of the $\mathrm{Moon}^{7,8}$ (Fig. 2). Recent isotopic evidence ${ }^{9}$ now dates this event at as a much as 100 million years after the origin of the Solar System. Many features of the event would also apply to earlier non-lunar-forming events, except that those would have been less extreme. The impact origin of the Moon was once a controversial idea, but it has gradually been accepted for two reasons: the lack of a realistic alternative, and growing evidence for its compatibility with the data - isotopic data in particular. Particularly importantly, it is thought to set the stage for Earth's subsequent evolution.

The lunar-forming collision plausibly involved the oblique impact of a Mars-mass planetary embryo (10\% of Earth's mass) with the $90 \%$ complete Earth. The impact velocity would probably have been dominated by the infall into the mutual gravity field, and most of this energy would have been converted into heat. Unlike energy, angular momentum is much more nearly conserved throughout geological time, and this kind of impact explains well the current angular momentum of the Earth-Moon system. The mean temperature rise of Earth resulting from this collision can be estimated as $\Delta T \approx 0.1 G M / R C_{\mathrm{p}} \approx 4,000 \mathrm{~K}$, where $G$ is the gravitational constant, $M$ and $R$ are Earth's mass and radius, respectively, and $C_{\mathrm{p}}$ is the specific heat of rock. Previous impacts would have heated Earth up to a hot, nearly isentropic state (a state in which entropy is nearly uniform with depth) close to, or partly in excess of, melting. Convective cooling below the freezing point is inefficient, so the state immediately before impact is hot, except perhaps right at the surface.

We expect that the impact heating would have been uneven because the various parts of Earth would be shocked to differing extents, but the immediate post-giant-impact state would relax to a very hot configuration, in which all or most of the rock and iron is in molten form and some silicate (perhaps even tens of per cent) is in vapour form. In most simulations of this kind of impact, a disk forms, derived mostly from the impacting body. For the expected radiating surface area and radiating temperature $(\sim 2,000 \mathrm{~K})$, the cooling time to remove about half of the impact energy is around 1,000 years, perhaps somewhat shorter for the disk. This is a very short period relative to the time between major collisions, but a very important one. During this short period, the Moon forms, most of the core of the projectile merges with the core of the proto-Earth, some of the pre-existing Earth's atmosphere may be blown off, and a significant part of the deep, initially molten, mantle 
of the Earth will freeze without having the opportunity to differentiate (because the crystals are advected vigorously by the turbulent convective motions that accompany the cooling).

The Moon probably did not form immediately after the giant impact, even though orbital times for material placed about Earth are less than a day. Instead, it seems to be necessary to wait for hundreds to thousands of years, the timescale of disk cooling, as it is thought likely that the Moon did form completely molten. For reasons not fully understood, the need to cool the disk is of greater importance than the shorter timescales of dynamical evolution. Perhaps lunar formation should not be thought of as disconnected from the provenance and evolution of the deep Earth. The reason is that, after the giant impact, some exchange of material may have taken place between Earth and the disk, aided by the vigorous convection of both the liquid and vapour parts of each and the presence of a common silicate atmosphere. This picture of rapid exchange makes the disk more Earth-like, rather than like the projectile that was responsible for its formation. The picture was originally motivated by a desire to understand the remarkable similarity of Earth and Moon oxygen isotopes ${ }^{8}$ but also finds support in tungsten ${ }^{9}$ and possibly silicon ${ }^{10}$ isotopic evidence. However, we do not yet have a fully integrated model of lunar formation that is dynamically satisfactory as well as chemically acceptable.

\section{Core formation}

The core-formation events (one event per giant impact) are particularly important because core formation is the biggest differentiation process of Earth: it involves one-third of Earth's mass and a large energy release, because the iron is about twice as dense as the silicates. To a substantial extent, it also defines the composition of Earth's mantle. In the immediate aftermath of a giant impact, we expect a substantial part of the core of the projectile to be emulsified with the molten mantle of the pre-impact proto-Earth. The core and mantle materials are thought to be immiscible (like water and oil) despite the very high temperatures, perhaps as high as $10,000 \mathrm{~K}$ for some of the material. If the material is mixed down to a small scale (perhaps even to the point where there are centimetre-sized droplets of iron immersed in the liquid silicate) then the iron and silicate can chemically and thermally equilibrate at high temperature and pressure (Fig. 3a). The composition of the core and the iron content of the mantle were presumably set during these equilibration episodes. The silicon and hydrogen contents of the mantle may also be affected by this equilibration, as both are soluble in iron at high pressure and temperature. These elements are particularly significant: silicon content affects the mineralogy of Earth's mantle, and the fate of hydrogen may have much to say about the total water inventory of Earth at this early epoch and the flow of mantle rocks. However, much of Earth's water may have been delivered later.

It is likely that some of the projectile iron is not mixed down to the smallest scales but instead finds its way to the core just hours after the impact (Fig. 3b). This iron will not equilibrate, either thermally or chemically, and it thus carries a memory of previous core-forming events at earlier times in smaller bodies (the embryos discussed earlier). The emerging picture is a complex one in which we should not expect the core or mantle of Earth to have a simple chemical relationship that involves the last equilibration at a particular pressure and temperature, but rather to have been formed under a range of thermodynamic conditions involving a number of significant events at different times ${ }^{2,11}$.

Earth's atmosphere at the time of a giant impact might have been mostly steam and carbon dioxide $\left(\mathrm{CO}_{2}\right)$ - probably both were important. It is possible, but not certain, that a large part of the atmosphere was blown away immediately after the giant impact. Water vapour is, however, much more soluble than $\mathrm{CO}_{2}$ in magma, so that even if the atmosphere were ejected into space, outgassing from the underlying magma ocean would replenish much of it. An important feature of water vapour is that it has a strong greenhouse effect, and that may have allowed the retention of an underlying magma ocean, even for the long periods between giant impacts. However, this type of atmosphere can rain out if there is insufficient energy supplied to its base (sunlight alone is insufficient) and, as a consequence, any steam atmosphere may collapse on a geologically short timescale, leading to an Earth surface that is actually cool (able to have liquid water) even while the interior is very hot.

\section{Mantle differentiation}

The mantle of the post-giant impact Earth will cool very fast at first ${ }^{12}$, limited only by the black-body radiation that can escape from the top of the transient (initially silicate vapour) atmosphere. The thermal structure of the mantle is expected to be close to isentropic because that is the state of neutral buoyancy and therefore the state preferred by convection, provided that viscosity is low. The nature of the freezing within this convecting state is of great importance and is thermodynamically determined. Many materials have the property that if they are squeezed isentropically, they undergo freezing even as they get hotter. Equivalently, they melt if they are decompressed isentropically from a frozen but hot, high-pressure state. The former correctly describes the freezing of Earth's solid inner core (the hottest place in Earth, yet frozen) whereas the latter correctly describes the melting responsible for the generation of basaltic magma, the dominant volcanism on Earth and most voluminously expressed at the low mantle pressures immediately beneath mid-ocean ridges. Recent work $^{13,14}$ suggests that this picture may not apply for the deeper part of Earth's mantle, so that freezing may begin at mid-depths.

Even so, there will eventually come a point (perhaps as soon as a few thousand years) after a giant impact when the bottom part of the mantle

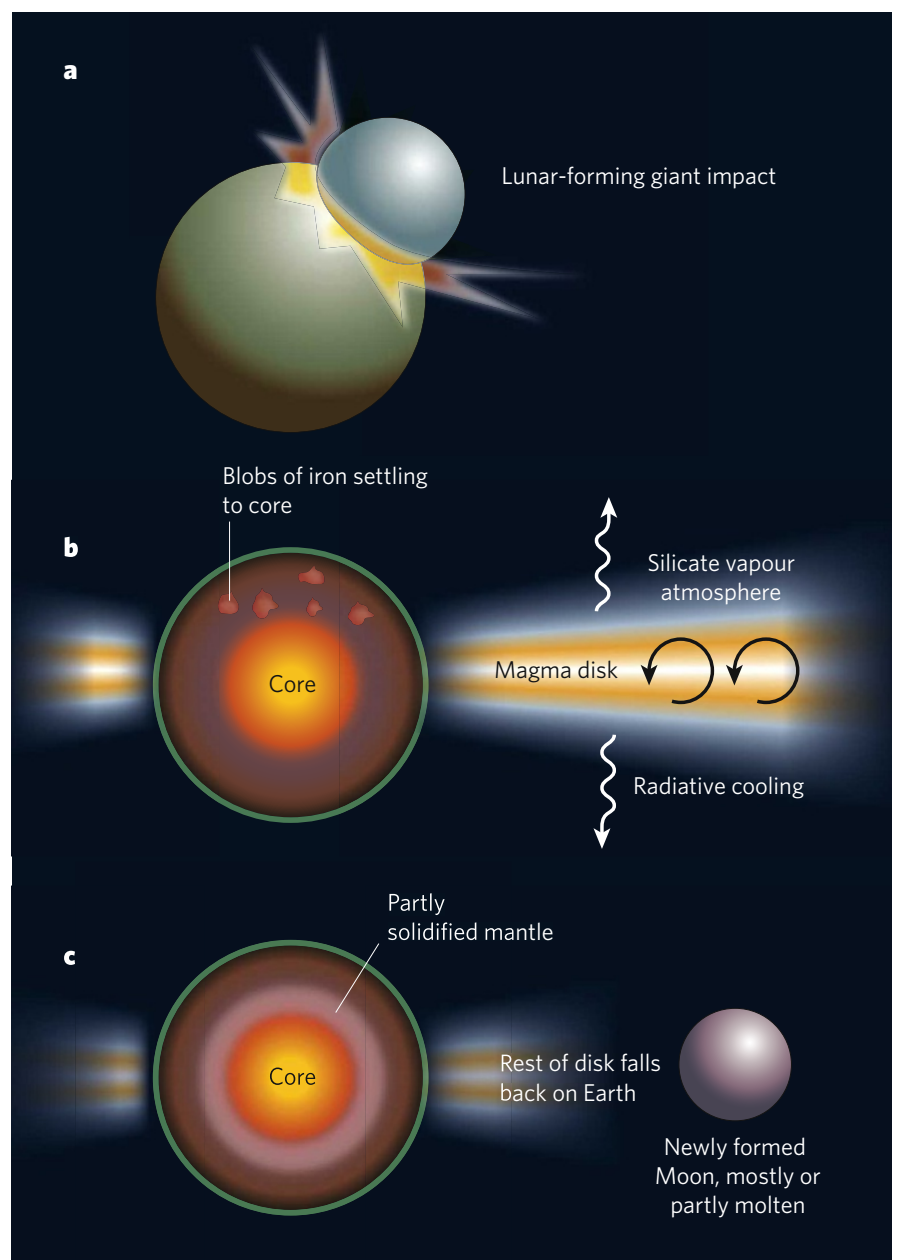

Figure 2 The effect on Earth of the giant impact that formed the Moon. a, A giant planetary embryo collides with the nearly complete Earth. b, A magma disk is in orbit about Earth, while blobs of iron from the planetary embryo settle down through the mantle to join the existing core. c, The outermost part of the magma disk coalesces to form the Moon as the result of radioactive cooling, while the rest falls back to Earth. Inside Earth, the mantle nearest the core has partly solidified, and the mantle might acquire a layered structure. 


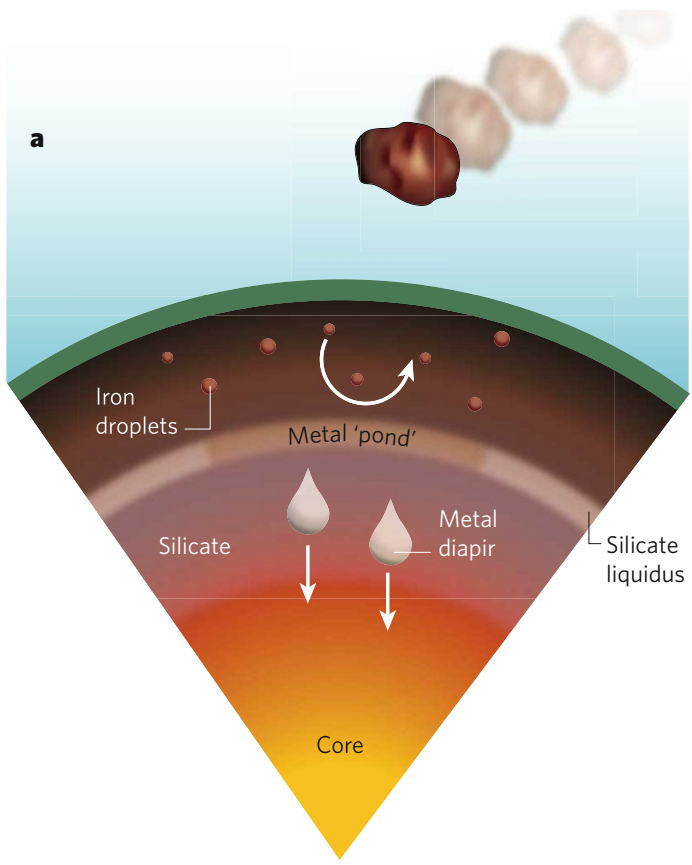

is mostly frozen. A very important question then arises: does the interstitial melt of this two-phase medium move up or move down under the action of gravity? It is very unlikely to be immobile. It is likely that it goes down (most probably because it is richer in iron than the coexisting solid), but in either case the mantle will differentiate internally into a layered structure (Fig. 4). This does not necessarily mean that Earth developed a primordial layering that has been preserved throughout geological time and is perhaps present still as part of the complex structure observed at the base of the mantle by seismologists and given by them the unromantic name of $\mathrm{D}^{\prime \prime}$ (see page 269). An early differentiation event for the silicate portion of Earth is favoured by some geochemists ${ }^{15}$, although, interestingly, it may have been earlier and it may have involved the formation of a primordial crust. It could perhaps be the cumulative consequence of giant impact events, a rare example of an Earth memory that even pre-dates the last giant impact.

The 'average' Earth surface environment during accretion may not have been very hot, even though there were undoubtedly short periods of time during which it was so hot that rocks were vaporized. These traumatic events reset the clock for subsequent evolution and emphasize the importance of the last such global event. Soon after the last global traumatic event, it may even be possible to have had rocks that survived throughout subsequent Earth history. Certainly, zircons - tiny, very resistant parts of rocks — have been dated back to $\sim 4.4$ billion years ${ }^{16}$, and it is not unreasonable to expect zircon discoveries that date back to within a few hundred million years of the lunar-forming impact. Zircons are not the same as hand specimens and rocks that can be studied in context (an intact structure, such as a surviving craton), but the gap is closing between the geological record as usually defined and the events that can only be dated through gross isotopic signals for Earth as a whole.

\section{Core memory}

The composition of Earth's core is different from pure iron-nickel and this is presumably because of the modest solubility of other elements, especially oxygen, silicon and sulphur. The simplest view of Earth's core is that it is a hot fluid cooled from above. Significantly, Earth's core has superheat: it is hotter than the temperature it would have been if liquid iron alloy coexisting with upper to mid-mantle silicates had sunk isentropically to the core. We can estimate this superheat by knowing the temperature at which iron alloys freeze at the known pressure of Earth near its centre and by the seismological determination of the size of its inner core. This superheat is currently about $1,000 \mathrm{~K}$ or so, and
Figure 3 | Two contrasting views of what might have happened during core formation. a, There is a magma ocean bounded below by a mostly solid lower region: the dispersed iron aggregates before descending to the core. $\mathbf{b}$, Some of the iron from the core of the projectile responsible for a giant impact is imperfectly mixed and descends to the core on a short timescale as distorted blobs hundreds of kilometres in diameter, without equilibration with the mantle. may initially have been in excess of 2,000 K. Unlike the mantle, the core cannot lose energy directly to the surface or to space and it is therefore likely that part of this superheat is a memory of the primordial Earth and may be telling us something about the specific processes responsible for core formation. Loss of primordial heat, together with the latent heat released as the inner core freezes, is potentially sufficient to maintain convection in the outer core over geological time, although even this is in some doubt given currently favoured values of the thermal conductivity of the core. In addition, buoyancy can be provided by the exclusion of part of the light elements from the inner core or perhaps from material exsolving from the outer core and attaching itself to the mantle.

Earth's magnetic field is generated by a dynamo: vigorous convection in the liquid, electrically conducting, outer core amplifies the existing magnetic field and thereby balances the tendency of the electrical current and associated fields to undergo decay. It is possible that these energy sources were insufficient to generate Earth's magnetic field even for the period when we know it must have existed ${ }^{17}$. A modest amount of radiogenic heat, most plausibly from the decay of ${ }^{40} \mathrm{~K}$, is a suggested solution to the shortfall. The experimental support for this is equivocal, but given the possibly high temperatures for part of the core-forming materials, it may be more difficult to keep things out of the core (that is, to avoid the core becoming too low in density) than to get them in! The amount of potassium needed would be modest and so it might not be apparent as a marked depletion of potassium in Earth's mantle relative to elements of similar volatility. The ability of Earth to generate a magnetic field may also be linked to the presence of an efficient mechanism for eliminating heat through the planet's surface. Plate tectonics is a particularly efficient mechanism.

\section{Plate tectonics and life}

We understand why Earth's mantle convects: there is no alternative mechanism for eliminating heat. However, we do not understand why Earth has plate tectonics. It is sometimes described as merely a property of the particular form that mantle convection takes on our planet, but this begs the question. Plate tectonics is neither mandatory nor common (there is no clear evidence of its existence on any other planet so far). Nonetheless, many think its presence is deterministic: given the specific parameters of present-day Earth, it is the behaviour expected, in the same sense that a physicist setting up a convection experiment on a layer of fluid heated from below need not be concerned about whether his chosen fluid was once a vapour or a solid. Even in this point of view, the presence of plate tectonics is history-dependent. For example, the amount and distribution of water may be important, as it is well established that water in rocks 


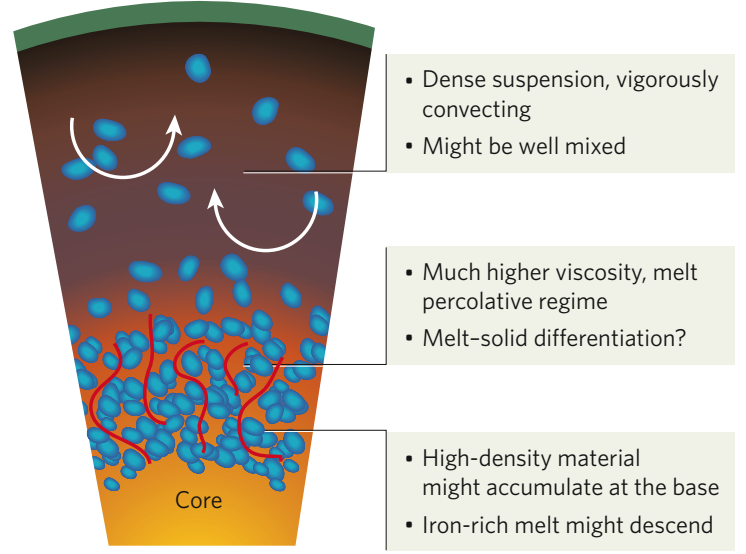

Figure 4 | Mantle cooling and differentiation during the later stages of a magma ocean. As the magma of the mantle cools, a stage is eventually reached at which dense iron-rich interstitial liquid (red) percolates through the solid matrix (blue) to accumulate just above the core.

has a major effect on their melting properties and response to stress. Earth's water budget is likely to be dependent on its history. The surface environment is profoundly influenced by the presence or absence of a plate-tectonic cycle, and that environment is, in turn, influencing the existence of life and is then affected by the presence of life. Everything affects everything else: the development of life on Earth is not likely to be disconnected from the composition of Earth's core.

\section{Where do we go from here?}

The remarkable advances over recent years and decades have been notable for their strongly interdisciplinary character, and some of this advance has come about through thinking of Earth as a planet and relating it to the environment in which it formed. Even so, the biggest challenge seems to require looking inside the planet: we need to understand better the phase relationships between Earth's constituents, the way in which mantle convection works and how to integrate this with plate tectonics, the connection between the deep Earth and our ocean and atmosphere, and the generation of Earth's magnetic field. The origin and development of life are also clearly questions for Earth science and will resist compelling answers until we have better characterized the thermodynamic, chemical and fluid dynamical environments. The deep Earth is deeply significant and also deeply informative for Earth's surface and all of Earth science.

David J. Stevenson is in the Division of Geological and Planetary Science, California Institute of Technology, Pasadena, California 91125, USA.

1. Udry, S. et al. The HARPS search for southern extra-solar planets $-X I$. Super-Earths (5 and $8 \mathrm{M}_{\text {}}$ ) in a 3-planet system. Astron. Astrophys. 469, L43-L47 (2007).

2. Halliday, A. N. \& Wood, B. J. in Treatise on Geophysics Vol. 9 (ed. Schubert, G.) 13-50 (Elsevier, Amsterdam, 2007).

3. Chambers, J. E. Planetary accretion in the inner Solar System. Earth Planet. Sci. Lett. 223, 241-252 (2004).

4. Raymond, S. N., Mandell, A. M. \& Sigurdsson, S. Exotic Earths: Forming habitable worlds with giant planet migration. Science 313, 1413-1416 (2006)

5. Ogihara, M., Ida, S. \& Morbidelli, A. Accretion of terrestrial planets from oligarchs in a turbulent disk. Icarus 188, 522-534 (2007).

6. Lissauer, J. J. \& Stevenson, D. J. in Protostars and Planets V (eds Reipurth, B., Jewitt, D. \& Keil, K.) 591-606 (Univ. Arizona Press, Tucson, 2007)

7. Canup, R. M. Dynamics of lunar formation. Annu. Rev. Astron. Astrophys. 42, 441-475 (2004).

8. Pahlevan, K. \& Stevenson, D. J. Equilibration in the aftermath of the lunar-forming giant impact. Earth Planet. Sci. Lett. 262, 438-449 (2007)

9. Touboul, M., Kleine, T., Bourdon, B., Palme, H. \& Wieler, R. Late formation and prolonged differentiation of the Moon inferred from W isotopes in lunar metals. Nature 450 , 1201-1209 (2007)

10. Georg, R. B., Halliday, A. N., Schauble, E. A. \& Reynolds, B. C. Silicon in the Earth's core. Nature 447, 1102-1106 (2007)

11. Rubie, D. C., Nimmo, F. \& Melosh, H. J. in Treatise on Geophysics Vol. 9 (ed. Schubert, G.) 51-90 (Elsevier, Amsterdam, 2007).

12. Solomatov, V. in Treatise on Geophysics Vol. 9 (ed. Schubert, G.) 91-119 (Elsevier, Amsterdam, 2007)

13. Stixrude, L. \& Karki, B. Structure and freezing of $\mathrm{MgSiO}_{3}$ liquid in Earth's lower mantle. Science 310, 297-299 (2005).

14. Mosenfelder, J. L., Asimow, P. D. \& Ahrens, T. J. Thermodynamic properties of $\mathrm{Mg}_{2} \mathrm{SiO}$ liquid at ultra-high pressures from shock measurements to $200 \mathrm{GPa}$ on forsterite and wadsleyite. J. Geophys. Res. 112, B06208 (2007).

15. Boyet, M. \& Carlson, R. W. Nd-142 evidence for early (>4.53 Ga) global differentiation of the silicate Earth. Science 309, 576-581 (2005).

16. Wilde, S. A., Valley, J. W., Peck, W. H. \& Graham, C. M. Evidence from detrital zircons for the existence of continental crust and oceans on the Earth 4.4 Gyr ago. Nature 409, 175-178 (2001).

17. Nimmo, F. in Treatise on Geophysics Vol. 9 (ed. Schubert, G.) 217-241 (Elsevier, Amsterdam, 2007).

Author Information Reprints and permissions information is available at npg.nature.com/reprints. Correspondence should be addressed to the author (djs@gps.caltech.edu). 